\title{
Surface mounting process analysis based on statistical management
}

\author{
Xu Dongxia, Wu Jiaofeng, Xu Juan \\ Guangdong Polytechnic of Environmental Protection Engineering, Foshan Guangdong, China, \\ 528216
}

Keywords: surface mount, statistical process, application

Abstract: In order to ensure the quality of surface mount work, the production technology of the placement technology is controlled within a reasonable range through statistical process control, and the overall benefit of the product is improved through reasonable control of the cost. Combined with the above, this paper elaborates on the concept of statistical process, and analyzes the rational application of statistical process control in surface mount technology, mainly from small batch production and production quality, including surface mount repeatability.

\section{Introduction}

Based on the many characteristics of surface mount technology, it is widely used in modern production, mainly electronic products. In the assembly process, it usually exhibits strong vibration resistance and strong solder joint tightness. It can be effectively used to reduce radiation in electronic products. For this advantage, we gradually found out in the later production process. The surface mount technology has the characteristics of cost saving and timeliness in the operation process, and the automation of the surface mount technology realizes the production efficiency effectively. Based on the characteristics of automation, we found that under the automated procedure, the quality of the products is often not guaranteed. We combine the statistical process control to control the quality of the products reasonably, and strive to minimize the quality of the products.

\section{The Description of Statistical Process Control (SPC)}

\subsection{Concept}

In order to facilitate the management process of the surface mount technology production process and reasonable control of product quality, it is necessary to take appropriate measures to rationally manage and supervise the whole process of production ${ }^{[1]}$. The production mode of modern products adopts large-scale mass production. In this process, the quality of the products will be subject to certain fluctuations due to the influence of the state of the production machinery. Starting from the surface mount dispensing process, there are seven steps, including several important steps such as welding and cleaning ${ }^{[2]}$. From the point of view of the sequential operation procedures of surface mount technology, the position of the dispensing and the working state of the dispenser will cause fluctuations in product quality. However, under normal circumstances, the operation of the machine will exhibit regular fluctuations. In addition to monitoring the quality, the function of statistical 
process control can be used to distinguish the causes of fluctuations in the process of mechanical application. Through the judgment of the program, the evaluation error of the fault is reduced, and the alarm is timely alarmed with abnormal fluctuations, so that the quality of the product can be reasonably and effectively controlled.

\subsection{Commonly Used Tools}

The tools commonly used in statistical process control are (PCA) and control charts. Their working principle is mainly to evaluate and judge the program specifications in the manufacturing process of products. Assuming that the fluctuations of the most affected fluctuations in the production process deviate, it is necessary to measure the indicators through the (PCI) capability index. In addition, another tool "control chart" is mainly used for image analysis by collecting data and sorting data. The position of the surface mount and the amount of glue to be dispensed should have strict numerical control. The control chart should be used to monitor the numerical variation existing in the production process, analyze the problem according to the data, and target it. control. When making the control chart, the following three quality indicators should be met, firstly the (CL) indicator, which requires the quality fluctuations to be within the scope of the indicator. Secondly, (UCL) and (LCL) control of these two indicators is in the range of the highest range, one in the range of the lowest range, the normal quality fluctuations in surface mount technology should be within the (CL) line in the general quality control process, according to the set value range of the control chart, it can accurately determine whether the process state is stable and whether the quality control is in a qualified state.

\subsection{Specific Practical Operations of Statistical Control Engineering}

\subsubsection{Determine the hardware selection of the statistical control project}

In order to effectively intervene the product with a large number of quality defects, it is necessary to start from the production process, timely troubleshoot the process failures that affect the quality problems, and control the failure problems in the shortest time to achieve product quality assurance [3]. In order to achieve this intervention control, the hardware of the statistical control system should be upgraded first, and a touch screen should be added on the side of the production process to receive data, input data, and realize data detection and storage.

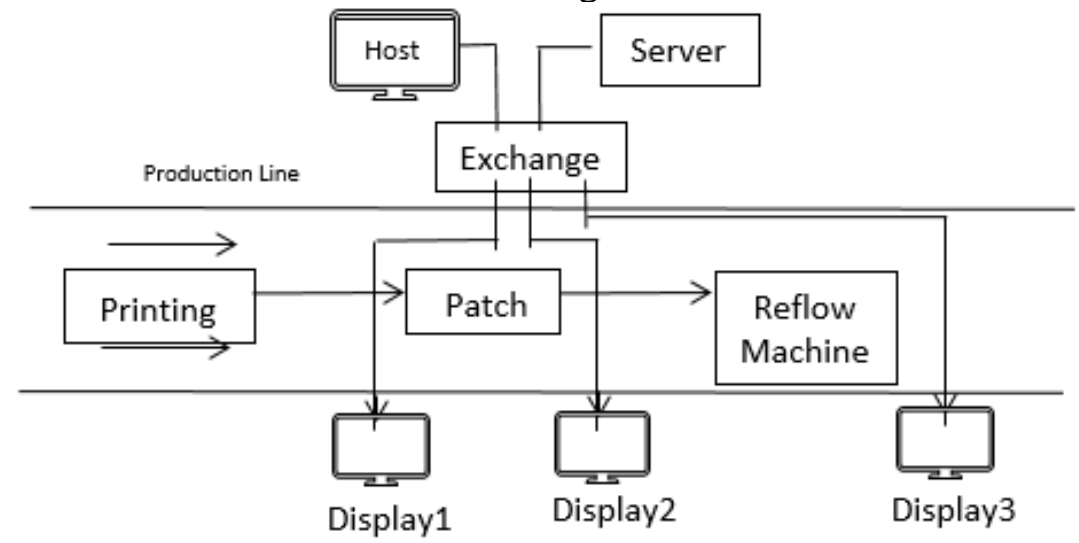

\subsubsection{The Design Concept of Software}

On the basis of perfect hardware facilities, the accuracy of data analysis should be ensured from the software settings. Under normal circumstances, statistical data control requires a relatively complex module to analyze a wide range of reasonable analysis. We elaborate from the production 
process: the product has a very high requirements on the production process in the production process. There are differences in the quality levels between different products. According to different types of products, the process should be properly set. In the placement process and the welding process in the surface mount application, it is necessary to determine the position of the placement machine in the SMT production line, and the process of curing and returning to the furnace requires melting the glue to firmly bond the components. On this basis, it is necessary to control the welding temperature and amplitude to prevent the tipping phenomenon occurring during the welding process.

\section{The Application Analysis of SPC in Surface Mount Technology}

\subsection{Used in Small Batch Production of Variety Diversity}

The most obvious feature of the progress of the meeting is the high development of electronic products. With the evaluation of the quality of electronic products and electronic products, the development trend of electronic products is smart and compact, in order to cater to human development, the quality of surface mount technology has become the most concerned issue. In order to effectively improve surface mount technology and improve the quality of surface mount, production technology combined with statistical process control has become a topic of research significance ${ }^{[4]}$. At present, the application of statistical process control is varied in a variety of applications and small batch production, and the control process cannot be easily identified. In this case, the accuracy of the control and the data of the control chart are prone to errors, so that the effect of the control is not obvious. Under the current situation, due to the environmental factors of the production process and the incomplete data volume of the control process, the correctness of the statistical effect cannot be evaluated. The small-scale production model does not have stable regularity, and the relevant data is not comprehensive enough, resulting in the realization of quality control is not obvious enough. The application of statistical process control has a great role in promoting the quality control in the production process. The use of historical data to establish a new modeling of the production process can effectively improve the cognitive process of statistical process control, and can be transformed against the theoretical system of cybernetics. Regional targets for quality monitoring.

\subsection{The Realization of the Function of the Production Process}

The function of statistical process control not only exists in the production process, but also can effectively manage and monitor the product purchase process. When surface mount technology is combined with statistical process control, in order to distinguish the standard value of the production part, the small part should be integrated into the overall reference object, the detection system should be set up, the standard parameters should be established according to the system, and the quality of the whole part should be substantive according to the standard parameters. At the same time, the function of statistical process control has the function of quality tracking. After monitoring the quality of the production process, the item is actively tracked in the form of bar code. Its function covers the monitoring of the production time of the item, and the circulation monitoring of the item, and the batch monitoring of the shipment of goods, in order to ensure the quality, the function of the statistical engineering control is comprehensive.

\subsection{Repeatability Index Used in SMT for Statistical Control Projects}

Set up a standard measurement point and establish the difference by extracting the number of points of the sample at this standard point. The repeat accuracy index refers to the use of the same 
measuring object at this standard point, and the same program is measured, and the standard values obtained by multiple measurements are summarized. The role of repeatability measurement is to use the statistical process engineering to conduct multiple tangible analysis of the quality standards of the manufacturing process, and to obtain the rules through multiple monitoring, in order to find out the quality failure points that do not meet the requirements in time, and to ensure the production quality, Guarantee the production standards of individual parts and improve the functional stability of the assembled parts.

\section{The Comprehensive Application Summary}

\subsection{Accurate Identification of Important Processes in the Operation Process}

The production process of surface mount technology has certain complexity, and there are related technical reports. However, in the production process, there must be a key production link. In surface mount technology, solder paste coating is an important operation link. Most of the placement defects are caused by mistakes in this link. The solder paste coating is the foundation for all subsequent processes. Therefore, in the process of product manufacturing, we should use experience to timely judge the key links in the product manufacturing process, start from the root cause, and avoid the quality problems in the production process as much as possible. In the statistical process engineering (SPC) application and surface mount technology (SMT), it is determined that the key process of the song is to monitor the production status of the key process, select the material for the solder paste coating process in Korea, the statistical process ( SPC) needs to collect the overall data in the process in detail, and make control charts based on the data at the same time. The control icons need to be compiled first, the limits are established, and the production status is based on the fluctuation range of the data. Judging whether it is normal or not, if the data fluctuates up and down within the normal range, it proves that the production process is normal and can smoothly carry out the follow-up work flow. The connection of this statistical project facilitates the supervision of the production process of most production enterprises in China. The growth of the national economy is inseparable from the development of the manufacturing industry. The production speed and renewal speed of various products should be proportional to the growth rate of the population. The production value of the manufacturing industry accounts for $35 \%$ of the total production value, and the productivity is huge. In the structure, production technology dominates. With the development of the social economy, the competitive position of the manufacturing industry is almost entirely based on the technological leadership of production technology, and sometimes the quality problems and process status in the manufacturing process may be neglected. Therefore, the application of SPC has social value.

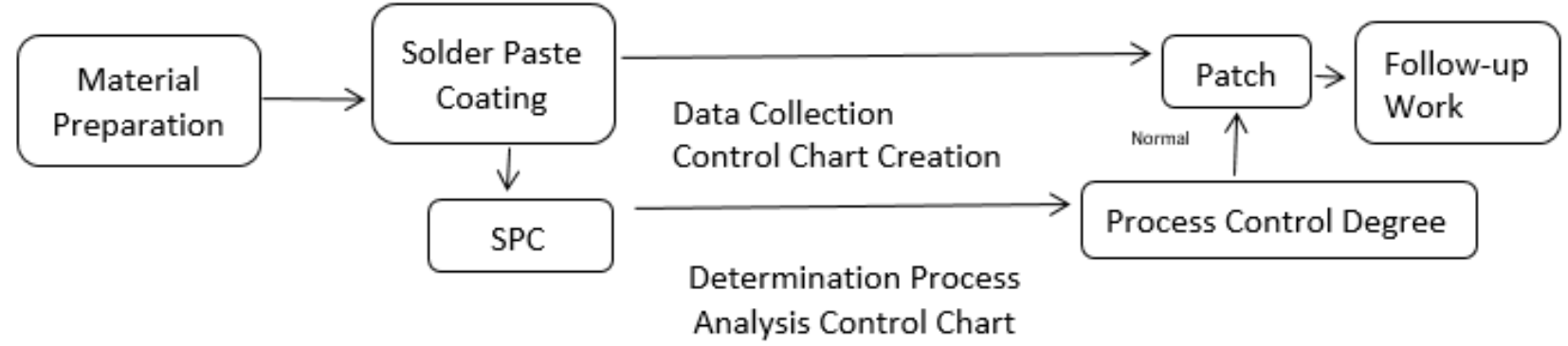

\subsection{Determining Data Statistics and Clarifying the Relationship between Variables}

We assume that the SPC application and the actual construction process, set the start time of the process, require that the sample be randomly selected once every 20 minutes, and that 4 sample is taken once, and 24 samples are specified ${ }^{[5]}$. We assume that $\mathrm{a}_{\mathrm{xy}}$ for data, use $x$ as a representation of 
a group, and use $y$ to indicate a certain data in the group.

$$
\begin{gathered}
\bar{a}_{x}=\frac{a_{x y 1+} a_{x y 2 \ldots+} a_{x y 4}}{4} \ldots \\
r_{x}=a_{\max }-a_{\min } \cdots
\end{gathered}
$$

\subsection{Design Control Chart for Data Analysis}

The establishment of the control chart mainly depends on the data model. Before the development of the new data, the existing data should be used for modeling. The preliminary calculation should be based on the following formulas, $x, y$, and sample number 24, respectively. Find the range and mean:

$$
\begin{gathered}
\bar{a}=\frac{\bar{a}_{x}+\bar{a}_{y 2-}+\bar{a}_{24}}{24}=z \cdots \\
\bar{R}=\frac{\bar{R}_{1}+\bar{R}_{2} \ldots+R_{24}}{24}=m \ldots
\end{gathered}
$$

According to the range and the mean value obtained by the above formula, it is necessary to calculate the corresponding data in the control chart by using the CL, $U C L_{X}, L C L_{X}$ of the control chart, where the coefficient is $\mathrm{A}_{1}$ :

$$
\begin{aligned}
& C L=\stackrel{=}{X}, \\
& U C L_{X}=\stackrel{=}{X}+A_{1} X \bar{R} \\
& L C L_{X}=\stackrel{=}{X}-A_{1} X \bar{R}
\end{aligned}
$$

According to the formula of the system, the specific calculation result is obtained, and then the control table is drawn by the control table coefficient, and the control table is observed. The total sample size of the sample is 24 , which means that there should be twenty points on the control table. Regularly distributed every 20 minutes. By observing the control chart, if the fluctuation of the point is within the central limit of the control line and exhibits a random state, then the production process is judged to be stable without failure or quality problems. Observing the distribution of the sample points, if there is a state of great fluctuation at a position approaching the upper and lower limits, and exhibiting irregularity, it is judged that there is a problem in the production process, and the alarm system is started to deal with the fault in time.

\subsection{Analyze Process Capability and Establish a Practice Control Chart}

Through the control chart, we must initially determine the state and stability of the production process. On this basis, we must classify the process according to the process capability, and we need to use the capability index judgment ${ }^{[6]}$. Through the control chart, the data analysis is carried out again, and the result is judged whether the total exercise of the process capability is sufficient, and the result is judged whether the production process and the process manufacturing process are reliably improved. The re-judgment of the control chart is to judge the fluctuation range of the manufacturing process, determine the stability of the manufacturing process and track and control it. According to the relevant technical report, the fluctuation pattern of the sampling points of the control chart is clarified. There are eight kinds in total. According to the known conventional fluctuation rules, the 
state of the overall manufacturing process is judged. The key link in the surface mounting technology is solder paste coating. Its working mechanism determines the operating standards for subsequent work. In order to reduce errors and improve quality, the solder paste coating procedure should use sample fluctuation analysis to make a rational judgment on the position and coating state of the solder paste coating, thus achieving true meaning. Correction, once the program is found to be abnormal, it should be immediately repaired, and the performance of the process program is improved for this fault, and the process is improved.

\section{Conclusion}

In order to meet the overall quality requirements of the production of the article, the quality of the product should be guaranteed from the production process to the delivery of the product batch, as well as the circulation and transportation after the product is finished. This is to improve productivity, save costs, and improve parts. In the future, the assembly activity is described in detail. Through detailed explanation of statistical engineering control, the specific application of statistical engineering in surface mount technology is described in detail, and the calculation formula is derived by using specific data to describe the production process and state judgment in the middle through control chart. Through the above technical examples, it is desirable to achieve the progress of the production process and effectively improve the product quality.

\section{Acknowledgement}

President Funding of Guangdong Polytechnic of Environmental Protection Engineering (KY201401002)

\section{References}

[1] Yan Sun, Gang Liu, Qinglei Jiang, et al. SPC Management and its Application in SMT Production Process [C]// 2014 China High-end SMT Academic Conference. 2014.

[2] Xiaonan Peng, Zhaohua Wu. Application of Statistical Process Control Technology in SMT Manufacturing[J]. Modern Surface Mount Information, 2003(4): 40-43.

[3] Ximing Yuan. Design of SMT Production Quality Online Analysis System Based on SPC[J].Measurement \& Control Technology, 2012, 31(11): 111-113.

[4] Hongxin Gou. How to Control SMT Process[J]. Modern Surface Mount Information,2007(2):51-53.

[5] Hui Zhang, Yuehua Gao, Bing Luo. Calculation of Repeatability Index in SMT Production SPC[J]. Electronic Quality, 2012(9): 53-55.

[6] Dan Zhang. Implementation of Quality Control Chart System and Its Application in SMT[J]. Electronic Quality, 2006(4): 39-41.

1) Xu Dong Xia (1978-),female, the Han nationality, Shangqiu people in Henan Province, master, associate professor, mainly engaged in electrical engineering and automation technology research.

2) Wu Jiao Feng (1978-),female, the Han nationality, Lvliang people in Shanxi Province, master, lecturer, mainly engaged in electrical engineering and automation technology research.

3) Xu Juan (1970-),female, the Han nationality,Tianmen people in Hubei Province, master, associate professor, mainly engaged in electrical engineering and automation technology research. 